\title{
Biologia reprodutiva de Pseudolaelia corcovadensis (Orchidaceae): melitofilia e autocompatibilidade em uma Laeliinae basal ${ }^{1}$
}

\author{
EDUARDO L. BORBA ${ }^{2,4}$ e PEDRO IVO S. BRAGA ${ }^{3}$
}

(recebido: 12 de março de 2003; aceito: 18 de setembro de 2003)

\begin{abstract}
Reproductive biology of Pseudolaelia corcovadensis Porto \& Brade (Orchidaceae): melittophyly and selfcompatibility in a basal Laeliinae). The floral biology, mating systems and phenology of Pseudolaelia corcovadensis (Orchidaceae), in the "Estação de Pesquisa e Desenvolvimento Ambiental de Peti", São Gonçalo do Rio Abaixo, Minas Gerais state was studied. This species flowers from April to September, with a higher availability of flowers in June and July. The flowers are dark-pink, strongly zygomorphic, and have osmophores and nectar-guides absorbing ultraviolet light. However, the flowers of P. corcovadensis do not present nectar and are pollinated by Bombus (Fervidobombus) atratus Franklin, 1913 (Hymenoptera: Apidae) by deceit. Apparently, the flowers do not form a model-mimic pair with other species in the community, but mimic a generalized melittophilous food-flower. As a consequence, visits are very rare and fruit set is low (18\%). Pseudolaelia corcovadensis is self-compatible and presents inbreeding depression in the early stages of development. The phylogenetic position of the genus Pseudolaelia and studies on floral biology in related genera suggest that melittophyly and selfcompatibility are basal characters in the subtribe Laeliinae, with subsequent adaptive radiation to pollination by hummingbirds, Lepidoptera, Diptera and other Hymenoptera.
\end{abstract}

Key words - melittophily, Orchidaceae, pollination, Pseudolaelia, reproductive biology

RESUMO - (Biologia reprodutiva de Pseudolaelia corcovadensis Porto \& Brade (Orchidaceae): melitofilia e autocompatibilidade em uma Laeliinae basal). Foi estudada a biologia floral, o sistema reprodutivo e a fenologia de Pseudolaelia corcovadensis (Orchidaceae), na Estação de Pesquisa e Desenvolvimento Ambiental de Peti, São Gonçalo do Rio Abaixo, Minas Gerais. A espécie floresce de abril a setembro, com pico de floração em junho e julho. As flores são rosa-escuro, fortemente zigomorfas, possuem osmóforos e guias de néctar que absorvem luz ultra-violeta. Porém, as flores de $P$. corcovadensis não possuem néctar, sendo polinizadas por Bombus (Fervidobombus) atratus Franklin, 1913 (Hymenoptera: Apidae) através de mecanismo de engodo. Aparentemente as flores não formam um par mimético com outra espécie na comunidade, mas mimetizam um modelo generalizado de flor melitófila que oferece recompensa. Como conseqüência, as visitas são muito raras e a frutificação é relativamente baixa (18\%). Pseudolaelia corcovadensis é autocompatível e apresenta depressão endogâmica nos estágios iniciais de desenvolvimento. O posicionamento filogenético do gênero Pseudolaelia, aliado a estudos de biologia floral em gêneros próximos, permite indicar que melitofilia e autocompatibilidade sejam características basais na subtribo Laeliinae, com posterior radiação adaptativa para polinização por beija-flores, Lepidoptera, Diptera e outros Hymenoptera.

Palavras-chave - biologia reprodutiva, melitofilia, Orchidaceae, polinização, Pseudolaelia

\section{Introdução}

As Orchidaceae constituem uma das maiores famílias de plantas, com aproximadamente 20.000 espécies reconhecidas (Dressler 1993). A família apresenta alguns dentre os mais complexos e intrigantes mecanismos de polinização conhecidos, sendo suas flores

\footnotetext{
1. Trabalho desenvolvido no Departamento de Botânica da Universidade Federal de Minas Gerais.

2. Universidade Estadual de Feira de Santana, Departamento de Ciências Biológicas, Rodovia BR 116, km 3, Campus Universitário, 44031-460 Feira de Santana, BA, Brasil.

3. Universidade Federal do Amazonas, Instituto de Ciências Biológicas, Departamento de Biologia, Av. General Rodrigo Otávio Jordão Ramos, 3000, Aleixo, Campus Universitário, 69077-000 Manaus, AM, Brasil.

4. Autor para correspondência: borba@gmx.net
}

polinizadas por diversos grupos de insetos (Hymenoptera, Diptera, Lepidoptera e Coleoptera) e por aves (van der Pijl \& Dodson 1966). Os Hymenoptera, principalmente as abelhas, têm sido reconhecidos como o grupo mais importante de polinizadores das orquídeas, responsável pela polinização de cerca de $60 \%$ das espécies, e a polinização por este grupo de insetos tem sido indicada como característica basal na família (van der Pijl \& Dodson 1966, Dressler 1981).

A subtribo Neotropical Laeliinae, com aproximadamente 1.500 espécies em cerca de 43 gêneros, é a segunda maior das Orchidaceae (Dressler 1993). As Laeliinae constituem um dos melhores exemplos de radiação adaptativa na polinização entre as orquídeas, sendo encontrados praticamente todos os principais grupos de polinizadores observados na família, exceto Coleoptera (van der Pijl \& Dodson 1966, 
Dressler 1981). Apesar dos dados serem escassos e esparsos, aparentemente a maioria dos gêneros da subtribo é polinizada por Hymenoptera, embora a maioria das espécies seja polinizada por Lepidoptera, isso em função do gênero Epidendrum, com cerca de 800 espécies, predominantemente polinizado por borboletas e mariposas (Dodson 1962, van der Pijl \& Dodson 1966).

O gênero Pseudolaelia Hoehne possui 8 espécies (incluindo o gênero monotípico Renata Ruschi), restritas aos estados de Minas Gerais, Rio de Janeiro, Espírito Santo e Bahia, normalmente epífitas sobre espécies de Velloziaceae em afloramentos rochosos dos campos rupestres ou afloramentos de granito/gnaisse das formações florestais (Pabst \& Dungs 1975, 1977, Withner 1993, Barros 1994). O gênero faz parte de um pequeno clado basal das Laeliinae (van den Berg et al. 2000), que inclui outros dois gêneros, Isabelia Barb. Rodr. (incluindo Sophronitella Schltr. e Neolauchaea Kränzl., de acordo com van den Berg \& Chase 2001) e Constantia Barb.Rodr.

Não existem informações sobre a biologia reprodutiva de espécies de Pseudolaelia e a única espécie basal de Laeliinae estudada até o momento é Constantia cipoensis Porto \& Brade (Matias et al. 1996), polinizada por abelhas do gênero Xylocopa (Anthophoridae, Xylocopinae).

No presente trabalho, são apresentados dados sobre a biologia reprodutiva, abordando biologia floral, sistema reprodutivo e fenologia, de Pseudolaelia corcovadensis Porto \& Brade, espécie epífita sobre Velloziaceae, ocorrente nos estados do Rio de Janeiro e Minas Gerais, e os resultados são discutidos em relação à evolução das síndromes e mecanismos de polinização em Laeliinae.

\section{Material e métodos}

Área de estudo - O trabalho foi desenvolvido na Estação de Pesquisa e Desenvolvimento Ambiental de Peti (EPDA-Peti), uma reserva particular pertencente à Companhia Energética de Minas Gerais (CEMIG). A EPDA-Peti possui 1.374 ha e se localiza nos municípios de São Gonçalo do Rio Abaixo e Santa Bárbara, na região mineradora de Minas Gerais (figura 1). A área possui relevo ondulado formado por serras dos contrafortes da Cadeia do Espinhaço, com altitudes variando de 603 a 808 metros. Na região, são encontrados latossolo vermelho-amarelo, solos litólicos e afloramentos rochosos de gnaisse. O clima é do tipo Cwa (Köppen 1948), tropical de altitude com verões frescos e estação seca bem definida (maio a setembro), e os meses de outubro a março úmidos. A hidrografia, pertencente à bacia do Rio Doce, é formada pelo Rio Santa Bárbara e pelo reservatório de água originado do seu represamento (Usina Hidrelétrica de Peti/CEMIG). Na região, são encontradas capoeiras, floresta estacional semidecidual secundária e vegetação sobre afloramento rochoso de gnaisse. A população de $P$. corcovadensis estudada situa-se no Morro do Cruzeiro $\left(43^{\circ} 22^{\prime} 10^{\prime \prime} \mathrm{W}\right.$ e $10^{\circ} 53^{\prime} 15^{\prime}$ 'S), com altitude de 808 metros (figura 1). No local, P. corcovadensis forma três subpopulações com vários indivíduos (cerca de 200), sempre como epífita em Vellozia albiflora Pohl (Velloziaceae).

Fenologia - Foram marcados 100 indivíduos de P. corcovadensis para acompanhamento de sua fenologia, sendo realizadas visitas mensais à população (quinzenais durante a floração), de março de 1993 a abril de 1994, sendo quantificados o número de pseudobulbos, folhas e orgãos reprodutivos produzidos. Foram considerados indivíduos conjuntos de pseudobulbos com conexão física pelos rizomas, tomando-se o cuidado de não marcar mais de um indivíduo de $P$. corcovadensis por indivíduo de V. albiflora, uma vez que em ramos muito velhos essa conexão pode desaparecer. Foi observado o número de inflorescências produzidas pelos 100 indivíduos marcados, sendo que 69 delas foram deixadas para polinização aberta, quantificando-se o número de flores e frutos produzidos.

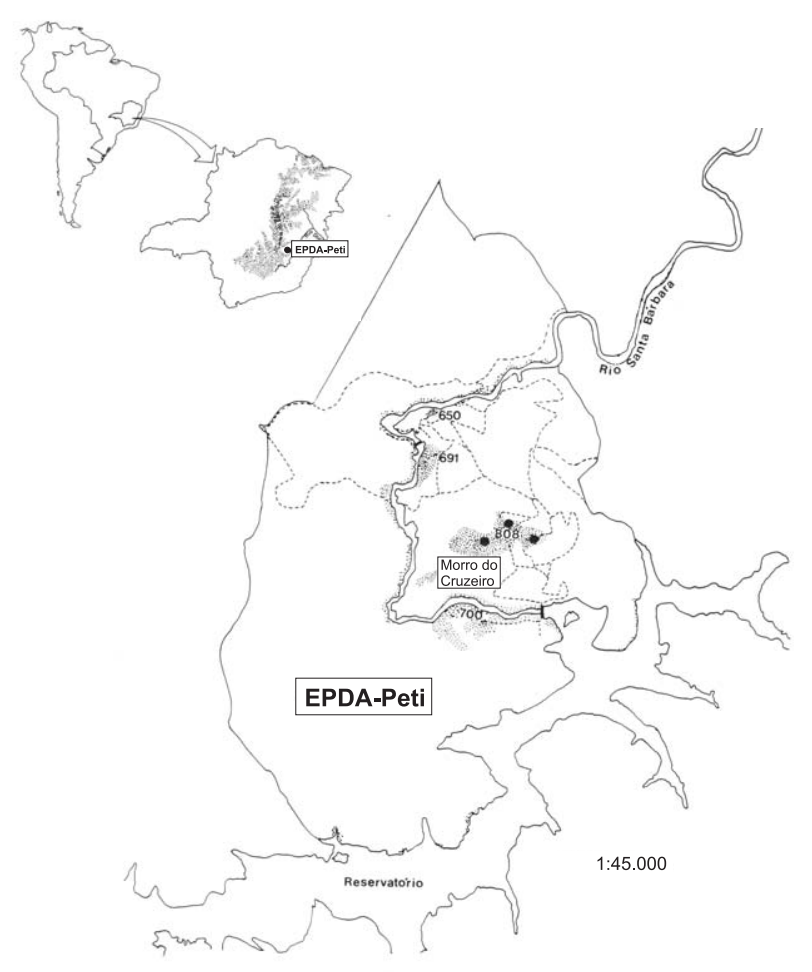

Figura 1. Localização da Estação de Pesquisa e Desenvolvimento Ambiental de Peti e local de ocorrência de Pseudolaelia corcovadensis ( $\bullet$ ).

Figure 1. Location of the "Estação de Pesquisa e Desenvolvimento Ambiental de Peti" and site of occurrence of Pseudolaelia corcovadensis (•). 
Biologia floral - Foram realizadas observações, inicialmente das 6:00 h às 19:00 h, para verificação da atividade dos visitantes florais, durante a floração de 1993. Devido à ausência de visitas no período vespertino e ocorrência de visitas no período matutino, durante a floração de 1994 o período de observações foi modificado para 6:00 h às 12:00 h, totalizando cerca de 100 horas de observação. Os visitantes foram coletados e enviados a especialistas para identificação. A ocorrência de nectários foi verificada através de dissecação das estruturas florais sob estereomicroscópio, e a presença de néctar foi analisada pelo exame das flores em campo, das 6:00 h às 19:00 h. A presença de osmóforos foi analisada mergulhando as flores em vermelho neutro a $1 \%$ por 10 minutos, lavando-as a seguir em solução de ácido acético glacial a 5\% (modificado de Vogel 1990). Os osmóforos diferenciam-se, permanecendo corados pelo vermelho-neutro. A presença de pigmentos que absorvem luz ultra-violeta foi verificada colocando-se as flores em atmosfera de hidróxido de amônio por 5 minutos (Scogin et al. 1977). As áreas contrastadas revelam a presença de flavonóides responsáveis pela absorção de luz ultra-violeta.

Sistemas de reprodução - Para análise dos sistemas de reprodução, 50 inflorescências, apenas com botões florais, foram cobertas por sacos confeccionados com tule para exclusão dos polinizadores. Após a antese, foram realizadas autopolinizações e polinizações cruzadas experimentais entre flores de um mesmo indivíduo (geitonogamia) e de indivíduos diferentes (xenogamia), sendo realizadas 35 polinizações para cada tratamento. As flores utilizadas nas polinizações cruzadas foram emasculadas. Uma amostra de 35 flores não polinizadas e não emasculadas foi utilizada como controle para verificação de agamospermia e autopolinização natural. Após a maturação dos frutos, foi avaliada a viabilidade dos embriões de uma amostra de 300 sementes por fruto formado a partir das polinizações experimentais $(n=19$; exceto para xenogamia, com $n=18$ ) e em frutos coletados na população em polinização natural $(n=20)$, empregando-se solução a $1 \%$ de cloreto 2,3,5-trifeniltetrazolium (Lakon 1949, Matias et al. 1996). Foi realizada análise de variância com um fator dos resultados de viabilidade, e as médias foram comparadas utilizando o Teste de Tukey, utilizando o pacote BioEstat 2.0 (Ayres et al. 2000).

Material testemunha de $P$. corcovadensis foi depositado no herbário da Universidade Federal de Minas Gerais (BHCB; E.L. Borba 4).

\section{Resultados}

Fenologia - Pseudolaelia corcovadensis apresenta crescimento vegetativo simpodial pela emissão de novos pseudobulbos. Eventualmente, um pseudobulbo pode emitir dois novos pseudobulbos, ocasionando uma ramificação do indivíduo, que leva à formação de duas frentes de crescimento. Ao início do estudo, os 100 indivíduos apresentavam um total de 142 frentes de crescimento (uma a quatro frentes por indivíduo). Durante o período de observação, ocorreram 45 novas ramificações. A emissão de pseudobulbos ocorreu de julho a janeiro. Apenas os cinco pseudobulbos terminais produziram novos pseudobulbos, sendo $72,1 \%$ deles produzidos pelo pseudobulbo terminal. Apenas $2 \%$ dos indivíduos não emitiram novos pseudobulbos. Dos 179 novos pseudobulbos, 11 (6,1\%) morreram no início do desenvolvimento.

A emissão de inflorescências ocorreu a partir de fevereiro, porém a antese das primeiras flores ocorreu apenas em abril, com a floração se estendendo até setembro. Foram produzidas 110 inflorescências em 94 (94\%) indivíduos e 110 (77,5\%) frentes de crescimento, sempre pelo pseudobulbo terminal. As inflorescências possuem de 35 a $110 \mathrm{~cm}$, com 13 flores em média, sendo produzido um total de 895 flores.

A frutificação se estendeu de abril a novembro, com o desenvolvimento dos frutos durando cerca de 130 dias. Ocorreu 18,3\% de frutificação, com 2,4 frutos produzidos em média por inflorescência. Na tabela 1 encontra-se um resumo dos eventos fenológicos e na tabela 2 os dados de produção de órgãos florais de P. corcovadensis.

Morfologia floral, néctar e emissão de odores Pseudolaelia corcovadensis possui flores eretas, ressupinadas por torção do pedicelo e ovário (figura 2A). Sépalas patentes, 13-19×7-8 mm, oblongas, membranáceas, róseo-escuras. Pétalas patentes, 13-21 $\times$ 6-8 mm, obovadas, membranáceas, róseo-escuras. Labelo ereto-patente, 12 -16 × 11-14 mm, adnado à base da coluna, membranáceo com centro levemente carnoso, trilobado, lobos laterais ligulados, lobo terminal obovado com margem levemente crenulada, magenta com calosidades brancas no centro e magenta-escuro na periferia. Coluna ereta, claviforme, 6-8 $\mathrm{mm}$ comprimento, branca na base e magenta no ápice. Antera apical com quatro pares de polínias.

O processo de antese durou aproximadamente 24 horas, estando as peças florais completamente livres no início da manhã do segundo dia, com a distensão completa do labelo e pétalas entre 7:00 h e 9:00 h. A antese de diferentes flores em uma inflorescência não ocorre em um mesmo dia, existindo até 3 a 4 flores disponíveis concomitantemente, uma vez que cada flor durou cerca de 4 a 5 dias.

As flores de $P$. corcovadensis possuem uma cavidade formando um cunículo com cerca de $6 \times 1,5 \mathrm{~mm}$, originado da adnação da base do labelo com a coluna (figura 2B), similar a uma cavidade nectarífera, 
Tabela 1. Quadro fenológico de Pseudolaelia corcovadensis na Estação de Pesquisa e Desenvolvimento Ambiental de Peti. (-) indica a ocorrência do evento fenológico, e (X) indica o período de maior intensidade do evento (no caso de frutificação, indica o período de deiscência dos frutos).

Table 1. Phenology of Pseudolaelia corcovadensis in the "Estação de Pesquisa e Desenvolvimento Ambiental de Peti". (-) indicates the occurrence of the phenologic event, and $(\mathrm{X})$ indicate the period of highest intensity of the event (in the fruiting period, it indicates the season of fruit dehiscence).

\begin{tabular}{|c|c|c|c|c|c|c|c|c|c|c|c|c|c|c|}
\hline \multirow[t]{2}{*}{ Evento } & \multicolumn{10}{|c|}{1993} & \multicolumn{4}{|c|}{1994} \\
\hline & mar & abr & mai & jun & jul & ago & set & out & nov & $\operatorname{dez}$ & jan & fev & mar & abr \\
\hline Emissão de pseudobulbos & & & & 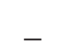 & - & - & $\mathrm{X}$ & $\mathrm{X}$ & $\mathrm{X}$ & $\mathrm{X}$ & - & - & & \\
\hline Emissão de folhas & & & & & & & - & - & $\mathrm{X}$ & $\mathrm{X}$ & - & - & & \\
\hline Presença de folhas & - & - & - & - & - & - & - & - & - & - & $\mathrm{X}$ & $\mathrm{X}$ & $\mathrm{X}$ & - \\
\hline Senescência foliar & $\mathrm{X}$ & $\mathrm{X}$ & $\mathrm{X}$ & $\mathrm{X}$ & $\mathrm{X}$ & $\mathrm{X}$ & $\mathrm{X}$ & - & - & - & $\mathrm{X}$ & $\mathrm{X}$ & $\mathrm{X}$ & $\mathrm{X}$ \\
\hline Floração & & - & - & $\mathrm{X}$ & $\mathrm{X}$ & - & - & & & & & & & - \\
\hline Frutificação & & & - & - & - & - & - & $\mathrm{X}$ & - & & & & & \\
\hline
\end{tabular}

porém não ocorreu produção de néctar em nenhum horário do dia. O teste do vermelho-neutro revelou a presença de osmóforos na posição mediana do labelo, coincidindo com as calosidades, porém não foi percebida a emissão de odores pelas flores em nenhum horário do dia. A reação com o hidróxido de amônio revelou a presença de flavonóides que absorvem luz ultra-violeta na metade proximal do labelo, coincidindo com a região de coloração branca e magenta-escuro nas calosidades, e no ápice da coluna.

Visitantes florais - A única espécie a visitar as flores de P. corcovadensis foi Bombus (Fervidobombus) atratus Franklin, 1913 (Hymenoptera: Apidae, Bombinae; figura 2C, D). Durante o período de observação, nos dois anos, apenas 7 visitas foram realizadas. As visitas ocorreram no início da manhã, entre 7:00 h e 9:00 h. A abelha pousou no lobo terminal do labelo, agarrando-se às margens com os dois primeiros pares de pernas. A seguir, ela colocou a cabeça entre o labelo e a coluna, inserindo a língua na cavidade do cunículo (figura 3). O inseto permaneceu apenas cerca de 3 segundos na flor, com a remoção do polinário ocorrendo quando o inseto recuou para alçar vôo da flor. Todo o polinário foi removido em uma única visita, fixando-se no rostro do inseto. Nas visitas realizadas, o polinizador visitou apenas uma flor na inflorescência, indo, em seguida, para fora da população ou eventualmente visitando uma flor em outro indivíduo distante. Não foi observada correlação entre a flor visitada na inflorescência e a sua idade. Bombus atratus nunca apresentou o comportamento de visitar seguidamente flores próximas (distâncias inferiores a cerca de 40 metros) na população de $P$. corcovadensis.
Ao longo de todo o período da manhã, foi observada intensa atividade de indivíduos de B. atratus visitando flores de Cuphea sp. (Lythraceae), nas quais coletam néctar. Esta espécie é muito abundante no local, possuindo flores tubulares de coloração rósea com cerca de $8 \mathrm{~mm}$ de comprimento e que produzem pequena quantidade de néctar, florescendo em sincronia e ocorrendo conjuntamente com os indivíduos de

Tabela 2. Produção de orgãos florais em 100 indivíduos de Pseudolaelia corcovadensis na Estação de Pesquisa e Desenvolvimento Ambiental de Peti. O número de flores e frutos refere-se a 69 inflorescências deixadas para polinização aberta.

Table 2. Flower and fruit set in 100 individuals of Pseudolaelia corcovadensis in the "Estação de Pesquisa e Desenvolvimento Ambiental de Peti". The flower and fruit numbers refers to 69 inflorescences left for open pollination.

\begin{tabular}{ll}
\hline Órgão & Quantidade \\
\hline Inflorescências emitidas & 110 \\
Indivíduos florescendo & $94(94 \%)$ \\
$\begin{array}{l}\text { Frentes de crescimento } \\
\quad \text { florescendo }\end{array}$ & $110(77,5 \%)$ \\
Tamanho das inflorescências & $70,7 \pm 13,1$ (amplitude: $35-110)$ \\
$\quad(\mathrm{cm})$ & \\
Flores produzidas & 895 \\
Flores por inflorescência & $13,0 \pm 3,6$ (amplitude: 6-28) \\
Frutos produzidos & $164(18,3 \%)$ \\
Frutos por inflorescência & $2,4 \pm 1,5$ (amplitude: $0-6)$ \\
Razão frutos/flores por & $18,4 \%$ (amplitude: $0-45 \%)$ \\
$\quad$ inflorescência & \\
\hline
\end{tabular}



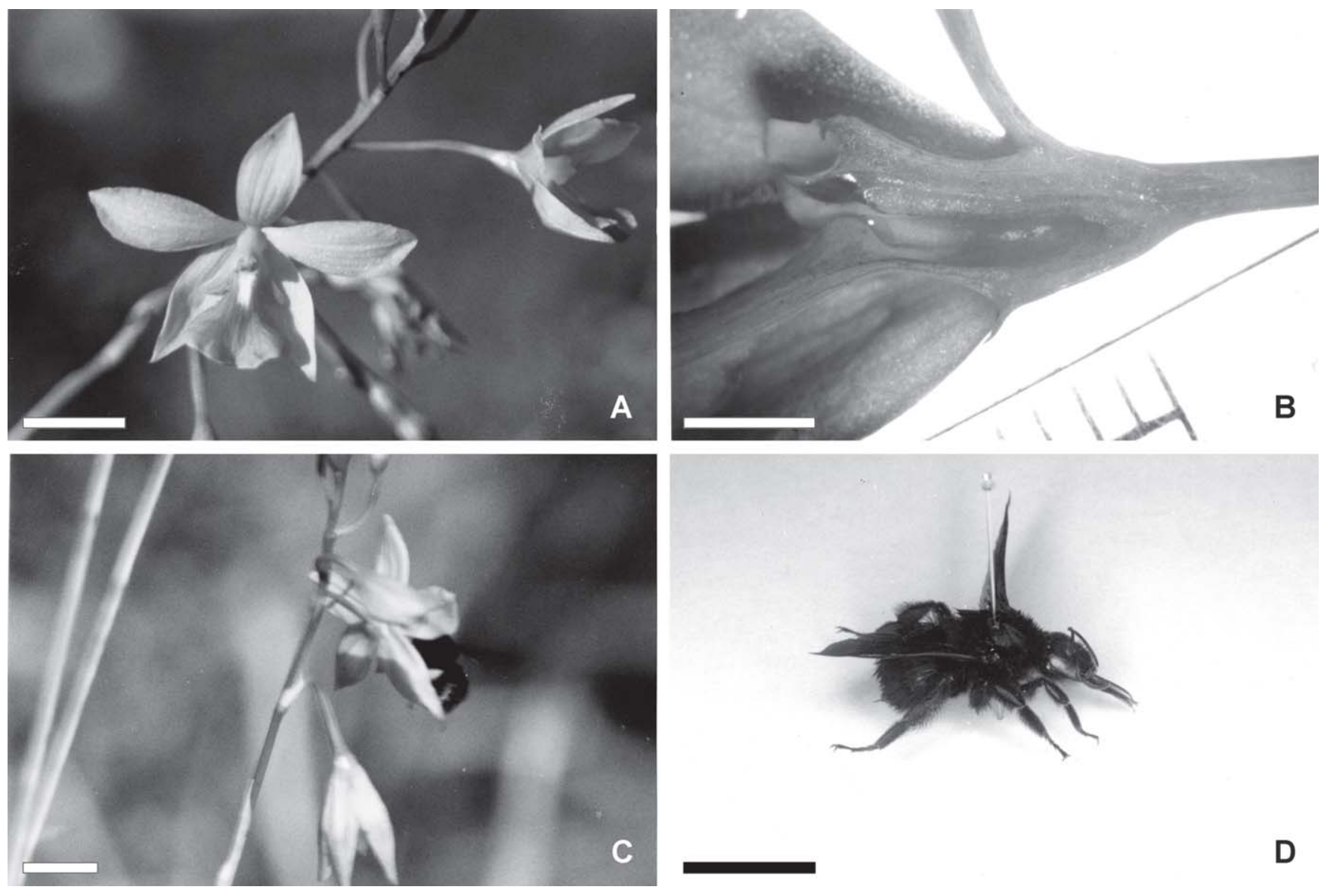

Figura 2. A. Flor de Pseudolaelia corcovadensis. B. Flor de P. corcovadensis em corte longitudinal, evidenciando a cavidade do cunículo. C. Bombus atratus visitando flor de $P$. corcovadensis. D. B. atratus, polinizador de $P$. corcovadensis. Barras $=$ $10 \mathrm{~mm}(\mathrm{~A}, \mathrm{C}), 3 \mathrm{~mm}(\mathrm{~B})$.

Figure 2. A. Flower of Pseudolaelia corcovadensis. B. Longitudinal section of flower of P. corcovadensis showing the cavity of the cuniculus. C. Bombus atratus visiting flower of $P$. corcovadensis. D. B. atratus, pollinator of $P$. corcovadensis. Bars $=$ $10 \mathrm{~mm}(\mathrm{~A}, \mathrm{C}), 3 \mathrm{~mm}(\mathrm{~B})$.

P. corcovadensis. As visitas de B. atratus às flores de Cuphea sp. são mais longas, podendo durar até cerca de 10 segundos. Bombus atratus possui um diferente comportamento de forrageamento na flores de Cuphea em relação às flores de $P$. corcovadensis, visitando, na primeira, normalmente o maior número de flores disponíveis em um indivíduo e se dirigindo, posteriormente, ao indivíduo mais próximo.

Sistemas de reprodução - Não ocorreu formação de frutos por apomixia ou autopolinização natural nas flores isoladas. Todas as flores polinizadas experimentalmente em todos os tratamentos desenvolveram frutos. Os frutos formados em todos os tratamentos amadureceram igualmente cerca de 130 dias após a realização da polinização.

A média da viabilidade das sementes (figura 4) em frutos formados por autogamia $(39,3 \%)$ não diferiu significativamente dos frutos formados por geitonogamia $(39,9 \% ; p=0,931)$, e ambas foram significativamente inferiores à dos frutos formados em xenogamia (55,7\%; $\mathrm{p}<0,01)$ e dos frutos formados em polinização aberta $(54,2 \% ; \mathrm{p}<0,01)$. As médias destes dois últimos tratamentos não diferiram significativamente entre si $(\mathrm{p}=0,782)$.

\section{Discussão}

O mecanismo de polinização encontrado em P. corcovadensis é amplamente distribuído na família Orchidaceae, denominado por Dressler (1981) como "key-hole flower", na qual a posição do polinizador é controlada pela pequena abertura que dá acesso à recompensa. Nesse mecanismo, a abertura mais ou menos tubular permite apenas a passagem da língua, 
bico ou probóscide do polinizador, que fica precisamente posicionado na flor, sendo o polinário depositado na face, como observado em $P$. corcovadensis, ou no bico ou probóscide do animal.

As flores de $P$. corcovadensis não oferecem recompensa, enganando o polinizador. Aparentemente, P. corcovadensis não apresenta mimetismo Batesiano, no qual a espécie formaria um par mimético com uma outra espécie na comunidade, mas mimetiza um modelo

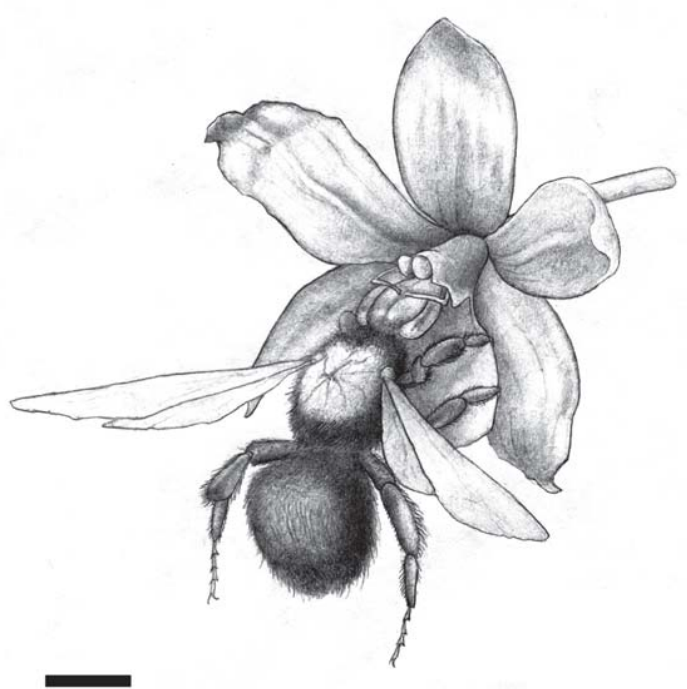

A

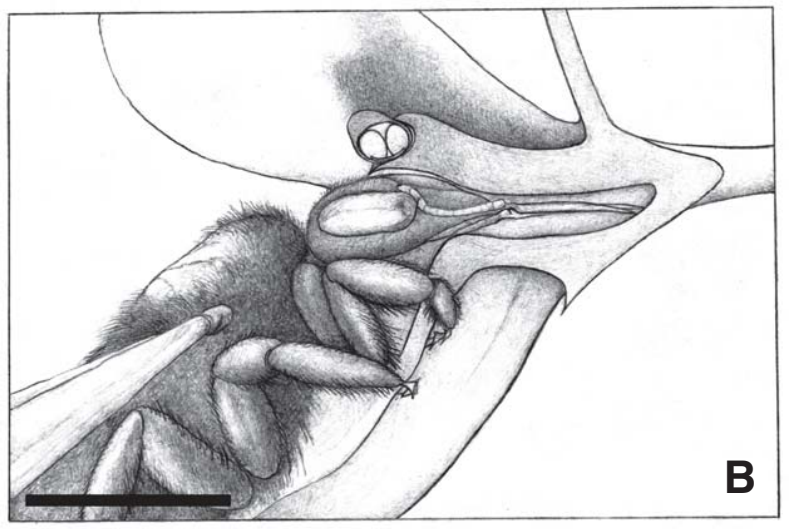

Figura 3. Esquema ilustrando o mecanismo de polinização em Pseudolaelia corcovadensis. A. Bombus atratus visitando flor de $P$. corcovadensis. B. Flor de P. corcovadensis em corte longitudinal $\operatorname{com} B$. atratus inserindo a língua na cavidade do cunículo. Barras $=3 \mathrm{~mm}$ (A), $5 \mathrm{~mm}$ (B).

Figure 3. Pollination mechanism in Pseudolaelia corcovadensis. A. Bombus atratus visiting flower of P. corcovadensis. B. Longitudinal section of flower of $P$. corcovadensis with $B$. atratus introducing its tongue in the cavity of the cuniculus. Bars $=3 \mathrm{~mm}$ (A), $5 \mathrm{~mm}$ (B). geral de flor melitófila que oferece recompensa, devido às suas características morfológicas, coloração, guias de néctar com absorção de luz ultra-violeta e presença de osmóforos. Esse tipo de mimetismo de um modelo generalizado é o mais comum em Orchidaceae, encontrado em 8.000 a 10.000 espécies na família (Ackerman 1981, 1986a, b, Dafni \& Calder 1987, Catling \& Catling 1991, Nilsson 1992, Johnson 1993), sendo o mimetismo Batesiano de ocorrência rara (Johnson 1994, 2000). Devido à capacidade de aprendizado dos polinizadores, especialmente das abelhas, flores de engodo recebem menor visitação e, conseqüentemente, apresentam menor frutificação do que espécies que oferecem recompensa, uma vez que, nas primeiras, os animais tendem a não retornar às flores (Ackerman 1975, 1981, 1983, Dafni \& Calder 1987, Braga 1977, Montalvo \& Ackerman 1987, Gill 1989, Nilsson, 1992, Rodrígues-Robles et al. 1992, Sabat \& Ackerman 1996, Borba \& Semir 1998, 2001). Este mecanismo seria uma exploração do comportamento instintivo de forrageamento dos insetos, especialmente de abelhas, sendo a maioria das visitas exploratórias por abelhas jovens, em busca de recursos alimentares ou por abelhas que estão explorando recursos adicionais durante um período de mudança na floração das espécies da comunidade (Ackerman 1981, 1983, 1986a, b, Little 1983, Nilsson 1992, Johnson 1993).

Entre as várias hipóteses sugeridas para explicar a evolução dos mecanismos de engodo em Orchidaceae, a mais amplamente aceita sugere que ele evolui como resultado de maiores taxas de polinização cruzada alcançadas, devido às maiores distâncias de vôo pelos polinizadores enganados entre visitas sucessivas a flores da espécie (Dressler 1981, Nilsson 1992). Essa hipótese é sustentada pelas observações realizadas em $P$. corcovadensis, onde o polinizador nunca visitou sucessivamente flores próximas, permanecendo pouco tempo em cada visita e indo normalmente embora da população, resultando em frutificação relativamente baixa. Em Orchidaceae, baixa frutificação tem sido sugerida como adaptativa em alguns casos, especialmente em espécies epífitas, que comumente ficam expostas a limitação de recursos. Nesses casos, seria mais importante garantir baixa frutificação com elevadas taxas de polinização cruzada do que elevada frutificação com grande percentual de frutos resultantes de cruzamentos que envolvem menor variabilidade (Montalvo \& Ackerman 1987, Gill 1989, Calvo 1990, Nilsson 1992, Ackerman \& Zimmerman 1994). 
Ackerman (1986a) sugere que polinização por engodo em Orchidaceae provavelmente evoluiu a partir de sistemas que oferecem recompensa. Constantia cipoensis, uma espécie pertencente ao mesmo clado basal de Pseudolaelia em Laeliinae (van den Berg et al. 2000), oferece néctar como recompensa aos seus polinizadores (Matias et al. 1996). Desse modo, não é claro qual das duas estratégias seria uma característica basal no grupo. Por outro lado, a distribuição do engodo entre as espécies de Laeliinae sugere que esta característica tenha surgido várias vezes na subtribo.

Polinização por espécies de Bombus ocorre em vários gêneros não relacionados de orquídeas, tendo surgido independentemente várias vezes na família (Dodson 1962, van der Pijl \& Dodson 1966, Thien \& Marcks 1972, Ackerman 1975, 1981, Mehrhoff 1983, Davis 1988, Catling \& Catling 1989, 1991, Singer \&

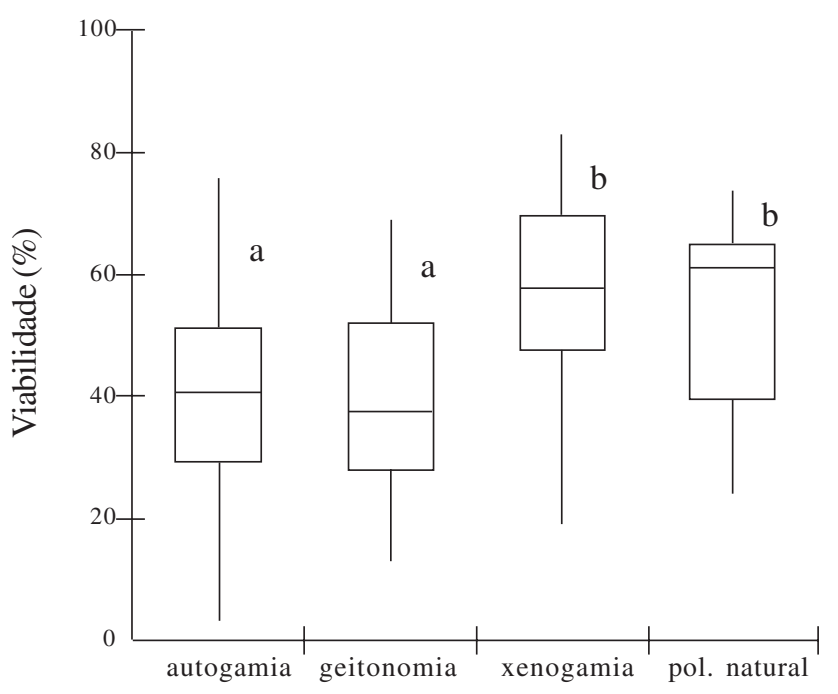

Figura 4. Box-plots da viabilidade percentual de sementes por fruto $(n=300)$ em polinizações experimentais simulando autogamia $(n=19)$, geitonogamia $(n=19)$ e xenogamia $(n=18)$ e de frutos formados naturalmente em polinização aberta $(n=20)$ em Pseudolaelia corcovadensis. A caixa compreende $50 \%$ dos dados e a linha central marca a mediana. Os traços superiores e inferiores são definidos pela amplitude dos interquartis.

Figure 4. Box-plots of percentual seed viability per fruit $(n=300)$ in experimental pollinations simulating autogamy $(n=19)$, geitonogamy $(n=19)$ and xenogamy $(n=18)$ and in naturally developed fruit in open pollination $(\mathrm{n}=20)$ in Pseudolaelia corcovadensis. The box comprises $50 \%$ of the data and the central line marks the median. Inner and outer fences are defined by interquartile ranges.
Sazima 1999). As Laeliinae apresentam ampla radiação adaptativa para diversos grupos de polinizadores, e, provavelmente, todas as síndromes de polinização surgiram várias vezes nas Laeliinae (van der Pijl \& Dodson 1966, van den Berg et al. 2000). A polinização por Bombus em Pseudolaelia e por Xylocopa (Hymenoptera: Anthophoridae, Xylocopinae) em Constantia (Matias et al. 1996) indica que melitofilia seja a condição basal em Laeliinae. A morfologia floral de outros gêneros basais, como Isabelia (incluindo Sophronitella e Neolauchaea), reforçam essa hipótese (veja van den Berg et al. 2000 para uma filogenia das Laeliinae).

Pseudolaelia corcovadensis é autocompatível, característica comum em Laeliinae (Stort \& Galdino 1984, Ianotti et al. 1987, Matias et al. 1996; mas, veja Adams \& Goss 1976, Goss 1977) e nas Orchidaceae em geral, onde a endogamia normalmente é evitada por barreiras mecânicas na flor ou etológicas do polinizador (van der Pijl \& Dodson 1966, Dressler 1981, Borba \& Semir 1999).

O percentual de sementes viáveis inferior em polinizações autogâmicas e geitonogâmicas indica a ocorrência de depressão endogâmica nos estágios iniciais de desenvolvimento (aborto de embriões) na população de $P$. corcovadensis, característica também freqüentemente observada em outras Laeliinae (Stort \& Martins 1980, Stort \& Galdino 1984, Matias et al. 1996) e outros grupos de orquídeas (Lock \& Profita 1975, Catling 1982, Borba et al. 2001) e que pode ser amplamente distribuída na família. A depressão endogâmica observada em $P$. corcovadensis é usualmente encontrada em populações de plantas predominantemente alógamas, mas com uma parte das polinizações realizados por endogamia (Stebbins 1974, Lande \& Schemske 1985, Schemske \& Lande 1985, Charlesworth \& Charlesworth 1987, Wiens et al. 1987, Husband \& Schemske 1996, Byers \& Waller 1999). Esta afirmação é reforçada pela similaridade na viabilidade das sementes entre as polinizações experimentais xenogâmicas e os frutos coletados na população formados em polinização aberta, que também sustenta a hipótese de evolução de engodo em Orchidaceae pelo favorecimento à alogamia.

Agradecimentos - Os autores agradecem a Júlio Antônio Lombardi e Fábio de Barros por sugestões nas versões iniciais e pelo encorajamento durante o desenvolvimento do trabalho. E.L. Borba agradece a bolsa de Produtividade em Pesquisa concedida pelo CNPq. 


\section{Referências bibliográficas}

ACKERMAN, J.D. 1975. Reproductive biology of Goodyera oblongifolia (Orchidaceae). Madroño 23:191-198.

ACKERMAN, J.D. 1981. Pollination biology of Calypso bulbosa var. occidentalis (Orchidaceae): a fooddeception system. Madroño 28:101-110.

ACKERMAN, J.D. 1983. Euglossine bee pollination of the orchid, Cochleanthes lipscombiae: a food source mimic. American Journal of Botany 70:830-834.

ACKERMAN, J.D. 1986a. Mechanisms and evolution of fooddeceptive pollination systems in orchids. Lindleyana 1:108-113.

ACKERMAN, J.D. 1986b. Coping with the epiphytic existence: pollination strategies. Selbyana 9:52-60.

ACKERMAN, J.D. \& ZIMMERMAN, J.K. 1994. Bottlenecks in the life histories of orchids: resources, pollination, population structure, and seedling establishment. In Proceedings of the 14th World Orchid Conference (A. Pridgeon, ed.). Her Majesty's Stationery Office, London, p.125-129.

ADAMS, R.M. \& GOSS, G.J. 1976. The reproductive biology of the epiphytic orchids of Florida III. - Epidendrum anceps Jacq. American Orchid Society Bulletin 45:488-492.

AYRES, M., AYRES JUNIOR, M., AYRES, D.L. \& SANTOS, A.S. 2000. BioEstat 2.0: aplicações estatísticas nas áreas das ciências biológicas e médicas. Sociedade Civil Mamirauá, Belém.

BARROS, F. 1994. Novas combinações, novas ocorrências e notas sobre espécies pouco conhecidas, para as orquídeas do Brasil. Acta Botanica Brasilica 8:11-17.

BORBA, E.L. \& SEMIR, J. 1998. Wind-assisted fly pollination in three Bulbophyllum (Orchidaceae) species occurring in the Brazilian campos rupestres. Lindleyana 13:203-218.

BORBA, E.L. \& SEMIR, J. 1999. Temporal variation in pollinarium size in species of Bulbophyllum: a different mechanism preventing self-pollination in Orchidaceae. Plant Systematics and Evolution 217:197-204.

BORBA, E.L. \& SEMIR, J. 2001. Pollinator specificity and convergence in fly-pollinated Pleurothallis (Orchidaceae) species: a multiple population approach. Annals of Botany 88:75-88.

BORBA, E.L., SHEPHERD, G.J. \& SEMIR, J. 2001. Selfincompatibility, inbreeding depression and crossing potential in five Pleurothallis (Orchidaceae) species. Annals of Botany 88:89-99.

BRAGA, P.I.S. 1977. Aspectos biológicos das Orchidaceae de uma campina na Amazônia Central. Acta Amazonica 7(Suplemento 2):1-89.

BYERS, D.L. \& WALLER, D.M. 1999. Do plant populations purge their genetic load? Effects of population size and mating history on inbreeding depression. Annual Review of Ecology and Systematics 30:479-513.

CALVO, R.N. 1990. Inflorescence size and fruit distribution among individuals in three orchid species. American Journal of Botany 77:1378-1381.
CATLING, P.M. 1982. Breeding systems of northeastern North American Spiranthes (Orchidaceae). Canadian Journal of Botany 60:3017-3039.

CATLING, P.M. \& CATLING, V.R. 1989. Observations on the pollination of Platanthera huronensis in southwest Colorado. Lindleyana 4:78-84.

CATLING, P.M. \& CATLING, V.R. 1991. A synopsis of breeding systems and pollination in North American orchids. Lindleyana 6:187-210.

CHARLESWORTH, D. \& CHARLESWORTH, B. 1987. Inbreeding depression and its evolutionary consequences. Annual Review of Ecology and Systematics 18:237-268.

DAFNI, A. \& CALDER, D.M. 1987. Pollination by deceit and floral mimesis in Thelymitra antennifera (Orchidaceae). Plant Systematics and Evolution 158:11-22.

DAVIS, R.W. 1986. The pollination biology of Cypripedium acaule (Orchidaceae). Rhodora 88:445-450.

DODSON, C.H. 1962. The importance of pollination in the evolution of the orchids of tropical America. American Orchid Society Bulletin 31:525-534, 641-649, 731-735.

DRESSLER, R.L. 1981. The orchids: natural history and classification. Harvard University Press, Cambridge.

DRESSLER, R.L. 1993. Phylogeny and classification of the orchid family. Cambridge University Press, Cambridge.

GILL, D.E. 1989. Fruiting failure, pollinator inefficiency, and speciation in orchids. In Speciation and its consequences (D. Otte \& J.A. Endler, eds.). Sinauer Associates, Sunderland, p.433-457.

GOSS, G.J. 1977. The reproductive biology of the epiphytic orchids of Florida V - Epidendrum difforme Jacq. American Orchid Society Bulletin 46:630-636.

HUSBAND, B.C. \& SCHEMSKE, D.W. 1996. Evolution of the magnitude and timing of inbreeding depression in plants. Evolution 50:54-70.

IANNOTTI, M.A., STORT, M.N.S. \& MARIN-MORALES, M.A. 1987. O sistema reprodutivo de Epidendrum rigidum Jacq. (Orchidaceae). Ciência e Cultura 39:641-644.

JOHNSON, S.D. 1993. Carpenter bee pollination of Herschelianthe graminifolia (Orchidaceae). Flora 188:383-386.

JOHNSON, S.D. 1994. Evidence for Batesian mimicry in a butterfly pollinated orchid. Biological Journal of the Linnean Society 53:91-104.

JOHNSON, S.D. 2000. Batesian mimicry in the non-rewarding orchid Disa pulchra, and its consequences for pollinator behaviour. Biological Journal of the Linnean Society 71:119-132.

KÖPPEN, W. 1948. Climatologia con un estudio de los climas de la Tierra (Trad. P.R.H. Peres). Fondo de Cultura Economica, Mexico.

LAKON, G. 1949. The topographical tetrazolium method for determining the germination capacity of seeds. Plant Physiology 24:389-394. 
LANDE, R. \& SCHEMSKE, D.W. 1985. The evolution of selffertilization and inbreeding depression in plants. I. Genetic models. Evolution 39:24-40.

LITTLE, R.J. 1983. A review of floral food deception mimicries with comments on floral mutualism. In Handbook of experimental pollination biology (C.E. Jones \& R.J. Little, eds.). Van Nostrand Reinhold, New York, p.294-310.

LOCK, J.M. \& PROFITA, J.C. 1975. Pollination of Eulophia cristata (Sw.) Steud. (Orchidaceae) in Southern Ghana. Acta Botanica Neerlandica 24:135-138.

MATIAS, L.Q., BRAGA, P.I.S. \& FREIRE, A.G. 1996. Biologia reprodutiva de Constantia cipoensis Porto \& Brade (Orchidaceae), endêmica da Serra do Cipó, Minas Gerais. Revista Brasileira de Botânica 19:119-125.

MEHRHOFF, L.A. 1983. Pollination in the genus Isotria (Orchidaceae). American Journal of Botany 70:1444-1453.

MONTALVO, A.M. \& ACKERMAN, J.D. 1987. Limitations to fruit production in Ionopsis utricularioides (Orchidaceae). Biotropica 19:24-31.

NILSSON, L.A. 1992. Orchid pollination biology. Trends in Ecology and Evolution 7:255-259.

PABST, G.F.J. \& DUNGS, F. 1975. Orchidaceae Brasiliensis, v.1. Kurt Schmersow, Hildeshein.

PABST, G.F.J. \& DUNGS, F. 1977. Orchidaceae Brasiliensis, v.2. Kurt Schmersow, Hildeshein.

RODRÍGUES-ROBLES, J.A., MELÉNDEZ, E.J. \& ACKERMAN, J.D. 1992. Effects of display size, flowering phenology, and nectar availability on effective visitation frequency in Comparettia falcata (Orchidaceae). American Journal of Botany 79:1009-1017.

SABAT, A.M. \& ACKERMAN, J.D. 1996. Fruit set in a deceptive orchid: the effect of flowering phenology, display size, and local floral abundance. American Journal of Botany 83:1181-1186.

SCOGIN, R., YOUNG, D.A. \& JONES, C.E. 1977. Anthochlor pigments and pollination biology: II. The ultraviolet patterns of Coreopsis gigantea (Asteraceae). Bulletin of the Torrey Botanical Club 104:155-159.
SCHEMSKE, D.W. \& LANDE, R. 1985. The evolution of selffertilization and inbreeding depression in plants. II. Empirical observations. Evolution 39:41-52.

SINGER, R.B. \& SAZIMA, M. 1999. The pollination mechanism in the 'Pelexia alliance' (Orchidaceae: Spiranthoideae). Botanical Journal of the Linnean Society 131:249-262.

STEBBINS, G.L. 1974. Flowering plants: evolution above the species level. Harvard University Press, Cambridge.

STORT, M.N.S. \& MARTINS, P.S. 1980. Autopolinização e polinização cruzada em algumas espécies do gênero Cattleya (Orchidaceae). Ciência e Cultura 32:1080-1084.

STORT, M.N.S. \& GALDINO, G.L. 1984. Self- and crosspollination in some species of the genus Laelia Lindl. (Orchidaceae). Revista Brasileira de Genética 7:671-676.

THIEN, L.B. \& MARCKS, B.G. 1972. The floral biology of Arethusa bulbosa, Calopogon tuberosus, and Pogonia ophioglossoides (Orchidaceae). Canadian Journal of Botany 50:2319-2325.

VAN DEN BERG, C., HIGGINS, W.E., DRESSLER, R.L., WHITTEN, W.M., SOTO-ARENAS, M.A., CULHAM, A. \& CHASE, M.W. 2000. A phylogenetic analysis of Laeliinae (Orchidaceae) based on sequence data from internal transcribed spacers (ITS) of nuclear ribosomal DNA. Lindleyana 15:96-114.

VAN DEN BERG, C. \& CHASE, M.W. 2001. Nomenclatural notes on Laeliinae - II. Additional combinations and notes. Lindleyana 16:109-112.

VAN DER PIJL, L. \& DODSON, C.H. 1966. Orchid flowers: their pollination and evolution. University of Miami Press, Coral Gables.

VOGEL, S. 1990. The role of scent glands in pollination. On the structure and function of osmophores (Trad. J.S. Bahatti). Smithsonian Institution Libraries, Washington.

WIENS, D., CALVIN, C.L., WILSON, C.A., DAVERN, C.I., FRANK, D. \& SEAVEY, S.R. 1987. Reproductive success, spontaneous embryo abortion, and genetic load in flowering plants. Oecologia 71:501-509.

WITHNER, C.L. 1993. The Cattleyas and their relatives, v.3. Schomburgkia, Sophronitis, and other South American genera. Timber Press, Portland. 